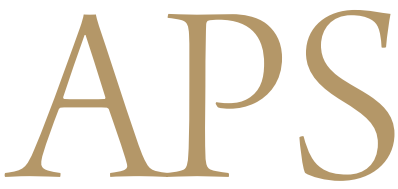

Archives of Plastic Surgery

\title{
Visible Perforating Lateral Osteotomy: Internal Perforating Technique with Wide Periosteal Dissection
}

\author{
Bong Il Rho ${ }^{1}$, In Ho Lee ${ }^{2}$, Eun Soo Park ${ }^{2}$ \\ ${ }^{1}$ Glovi Plastic Surgery, Seoul; ${ }^{2}$ Department of Plastic and Reconstructive Surgery, Soonchunhyang University Bucheon Hospital, \\ Soonchunhyang University College of Medicine, Bucheon, Korea
}

There are two general categories of lateral osteotomy techniques-the external perforating method and the internal continuous method. Regardless of which technique is used, procedural effectiveness is hampered by limited visualization in the surgical field. Considering this point, we devised a new technique that involves using a wide subperiosteal dissection and internal perforation under direct visualization. Using an intranasal approach, whereby the visibility of the intended fracture line was maintained, enabled a greater degree of control, and in turn, results that were more precise, and thus predictable and reproducible. Traditionally, it has been taken as dogma that the periosteum must be preserved, considering the potential for dead space and bony instability; however, under sufficient visualization of the surgical field with an internal perforating method, complete osteotomy with fully preserved intranasal mucosa could be conducted exactly as intended. This intact mucosal lining compensates for the elevated periosteum. Compressive dressing and drainage through a Silastic angio-needle catheter enabled the elimination of dead space. Therefore, precise, reproducible, and predictable osteotomy minimizing the potential for associated complications such as ecchymosis, that is, bruising owing to hemorrhage, could be performed. In this article, we introduce a novel technique for lateral osteotomy with improved visualization.

Keywords: Rhinoplasty / Osteotomy / Ecchymosis / Dissection
Correspondence: Eun Soo Park Department of Plastic and Reconstructive Surgery,

Soonchunhyang University Bucheon Hospital, Soonchunhyang University College of Medicine, 170 Jomaru-ro, Wonmi-gu, Bucheon 14584, Korea Tel: +82-32-621-5319 Fax: +82-32-621-5316 E-mail: peunsoo@schmc.ac.kr

The work was supported by the Soonchunhyang University Research Fund.

This article was presented at the 71st International Congress of the Korean Society of Plastic and Reconstructive Surgeons, November 2, 2013 in Seoul, Korea.

No potential conflict of interest relevant to this article was reported.

Received: 2 Apr 2015• Revised: 23 Sep 2015• Accepted: 19 Oct 2015

pISSN: 2234-6163 • elSSN: 2234-6171 • http://dx.doi.org/10.5999/aps.2016.43.1.88 • Arch Plast Surg 2016;43:88-92

\section{INTRODUCTION}

The lateral osteotomy is an essential procedure of cosmetic surgery, usually performed in order to narrow a wide nasal bone base, to resolve an open roof deformity after a humpectomy, or to refine the nasal bony pyramid. Surgeons can select from two basic methods to perform a lateral osteotomy: the external perforating method or the internal continuous method [1-6]. Ad- vocates of the external perforating method state that it is associated with less mucosal and periosteal trauma, superior bone stump stability from periosteal support, and a more controlled fracture $[1,2,6]$. Alternatively, advocates of the internal continuous method prefer the superior mobility of the bony vault and consistent narrowing of the nasal pyramid $[1,7]$. These standard lateral osteotomy methods are responsible for significant periorbital swelling and ecchymosis from injuries to the periosteum 
and to the intranasal mucosa attached to the osteotomy sites, which can be exacerbated by limited visualization in the surgical field. Despite some debate over the solutions to these problems, it is generally agreed that the ideal osteotomy method would produce precise, predictable, and reproducible results with fewer complications [6]. Patient reports of edema, mucosal tearing, ecchymosis, unfavorable and incomplete fractures, and unsatisfactory aesthetic outcomes are common after a lateral osteotomy is performed; this is especially true for cases in which visualization of the surgical field was impaired $[5,6,8,9]$. To retain the advantages of the existing techniques while simultaneously overcoming the disadvantage of surgical-field "blindness," we devised a simple and novel lateral osteotomy method that uses an internal perforating approach, whereby wide periosteal dissection is conducted under direct visualization. The aim of this article is to describe our experience with this new technique.

\section{IDEA}

\section{Surgical technique}

After the patient was sedated with propofol, a local anesthetic solution of $1 \%$ lidocaine with epinephrine $(1: 200,000)$ was injected into the areas along the lateral nasal sidewalls for vasoconstriction and subperiosteal hydrodissection. A V-shaped incision was made at the narrowest part of the columella and was continued through the vestibular skin along the caudal border of the lower lateral cartilage. The dissection was performed through the supraperichondrium to leave as much soft tissue on the skin flap as possible. From the keystone area, a subperiosteal elevation was then conducted with caution, so that the adjacent vessels were not injured. The subperiosteal dissection was continued to the lateral nasal wall and laterally along the frontal processes of the maxilla. We also performed part of the subperiosteal dissection more laterally than the intended osteotomy line just enough to ensure visibility of the surgical field. The nasal skin envelope was retracted and, to gain secured visualization of the osteotomy site, the lower lateral cartilages were also retracted (Fig. 1). Using a $2 \mathrm{~mm}$ straight, unguarded osteotome, we first performed a medial oblique osteotomy at $15^{\circ}$ to $20^{\circ}$. An internal perforating lateral osteotomy was then conducted along the planned osteotomy line, keeping the osteotome as perpendicular as possible to the bone. The osteotomy was started at the distal two-thirds point of the lateral side wall, while caution was taken to avoid injuring the Webster's triangle. In accordance with the low-to-high method, the osteotomy progressed upward. We were extremely careful to avoid damaging the intranasal mucosa just beneath the nasal bone to maintain the stability and vascularity of the bony stump with the internal perforating technique. The lateral osteotomy was therefore conducted more precisely under the security of direct vision, whereby the intranasal mucosa and adjacent vasculature including the lateral-nasal and angular vessels were preserved. The lateral osteotomy

\section{Fig. 1. Fully visualized surgical field}

The periosteum dissection was more lateral than the intended osteotomy by about 2-3 $\mathrm{mm}$ to secure direct visualization of the lateral osteotomy site. (A) Right (white arrow). (B) Left (white arrow).
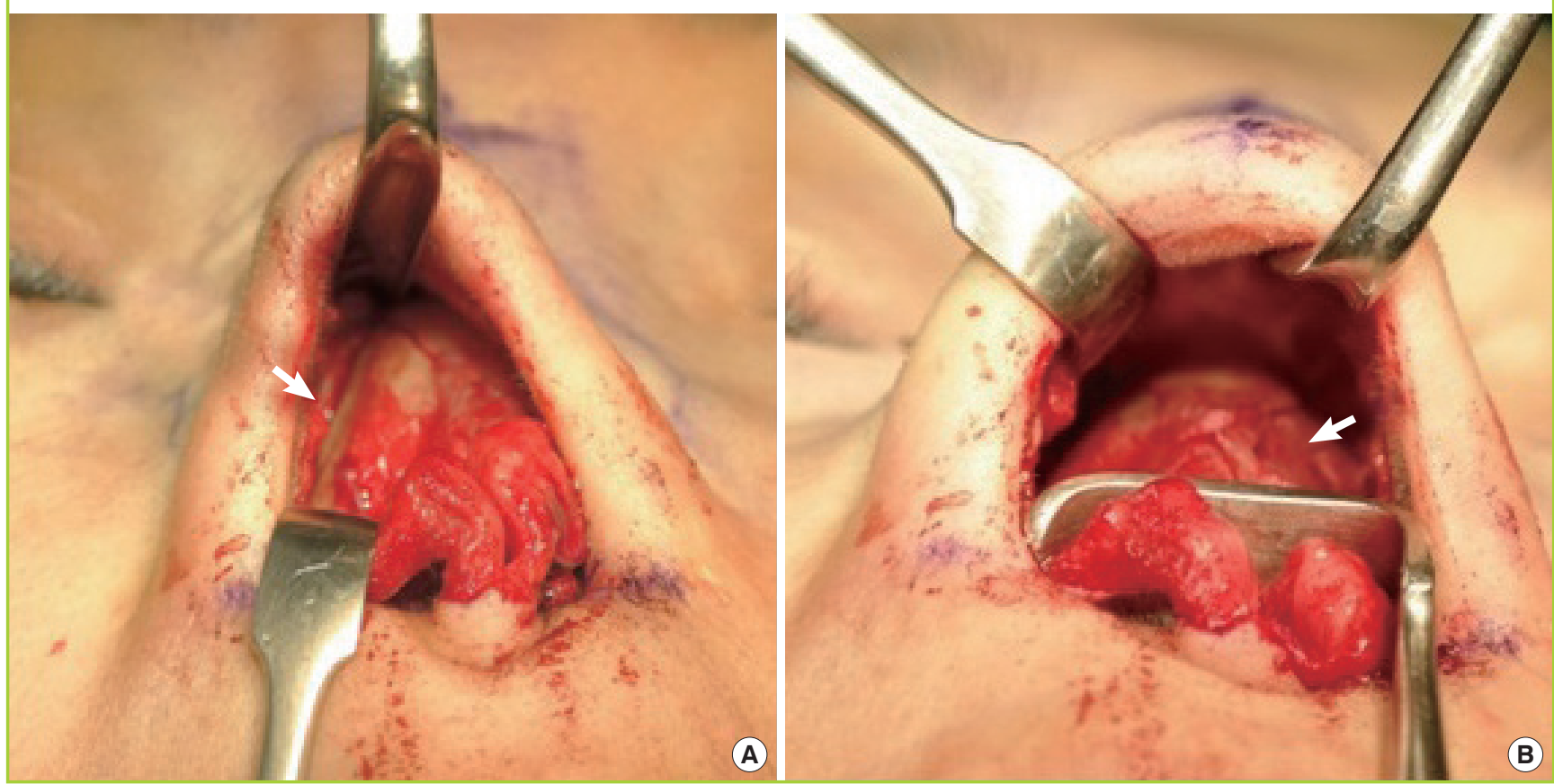
was usually terminated at the level of the point of meeting the medial osteotomy. After an identical procedure was performed on the contralateral side, gentle pressure was applied using a finger to induce an inward fracture. Notably, at this time, direct visualization was used to confirm a bony gap measuring about 1-2 $\mathrm{mm}$ inward and preserved intranasal mucosa (Fig. 2), enabling the accurate repositioning of the nasal bones in accordance with the surgical plan. Where necessary, the irregular bony edges

\section{Fig. 2. Internal mucosal lining as a splint for the bony stump}

The intended fracture line could be identified accurately under direct vision, and the intact intranasal mucosal lining acted as a splint, supporting the stability of the bony stump and the vasculature.

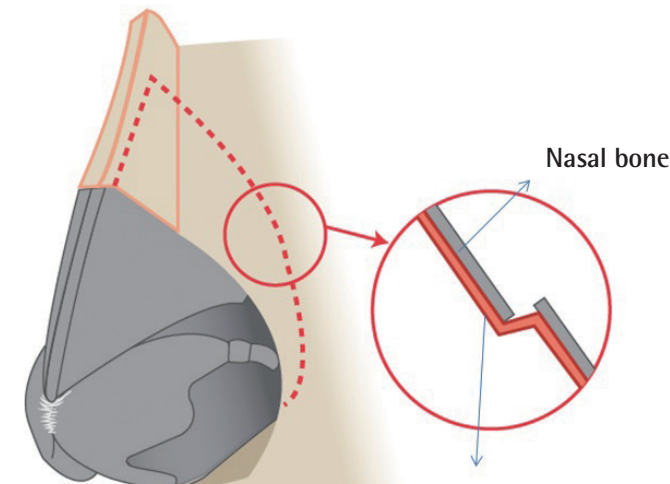

Intact intranasal mucosal lining

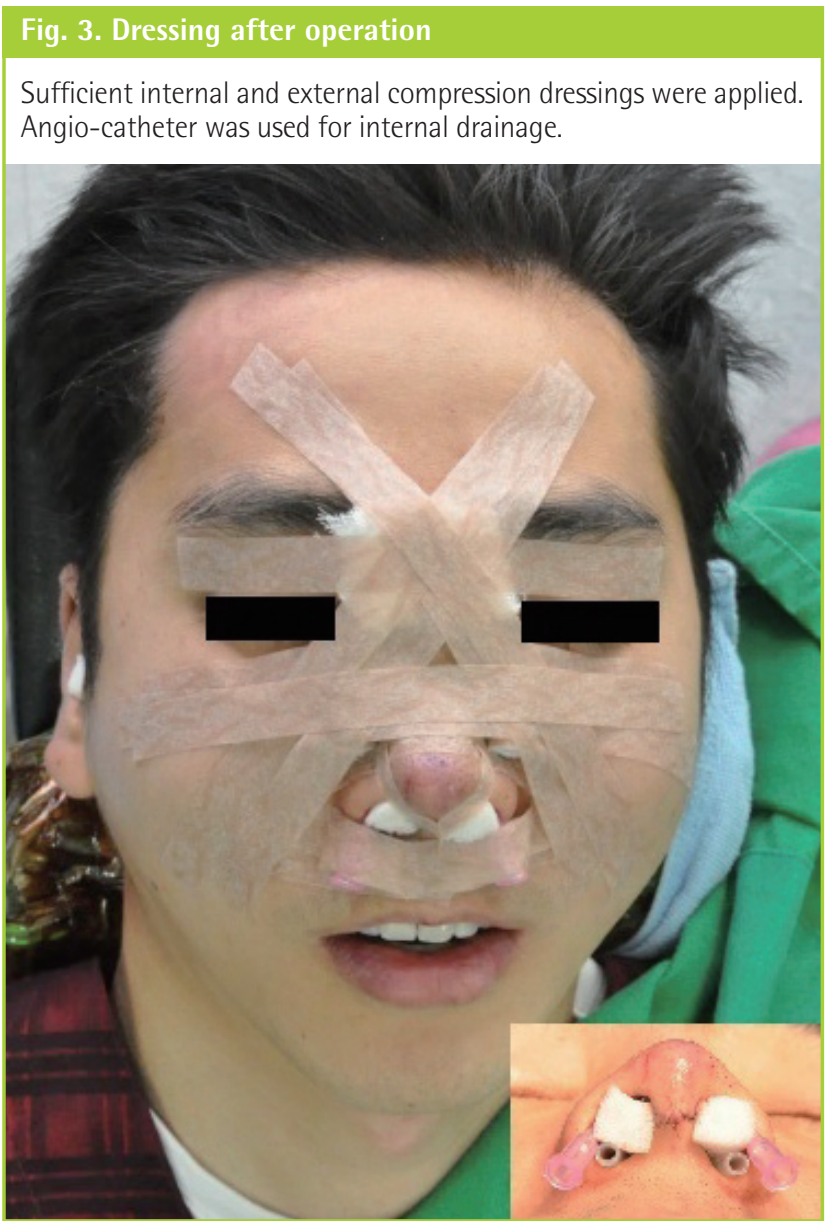

\section{Fig. 4. A 24-year-old female patient}

Preoperative (A) and four-month postoperative (B) views of a 24-year-old female patient who underwent primary rhinoplasty including a lateral osteotomy in which the internal visible perforating method was used, as well as humpectomy.
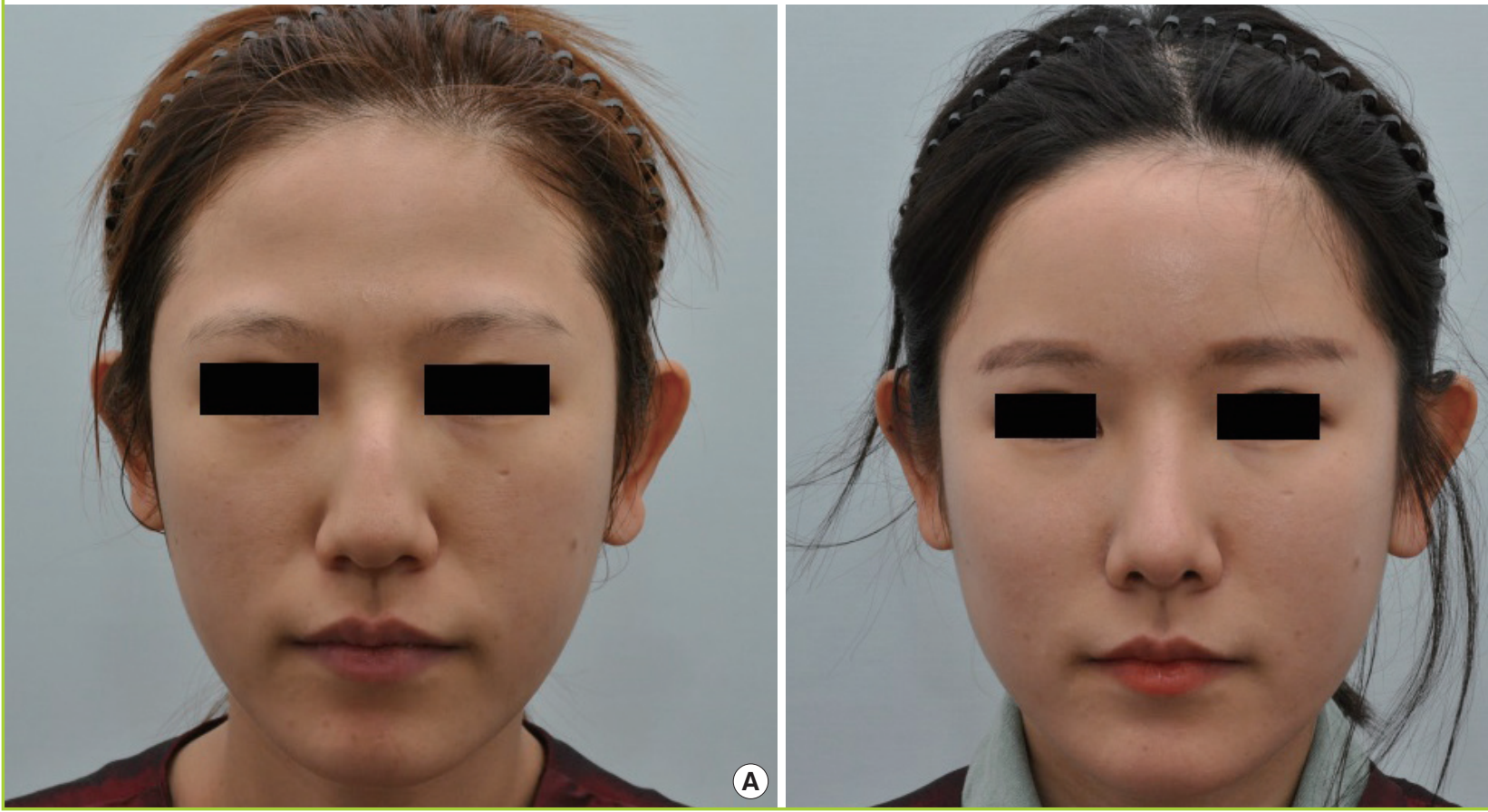
were rasped and other conventional techniques, such as tip plasty or augmentation, were performed. After an overall procedure was completed, compression dressing was applied and angioneedle catheter was introduced to the incision site for the purpose of drainage. A Silastic tube was inserted to maintain the airway and Merocel (Medtronic Inc., Minneapolis, MN, USA) was used to compress the operation site internally. With gauze pillows and gauze, external compression was applied across the radix, the lateral nasal wall, and both medial canthal areas until postoperative day one (Fig. 3).

\section{Case}

A 24-year-old female patient visited our clinic with a chief complaint of deviation, a hump, and broadness of the nasal dorsum. We performed a lateral osteotomy using our method, while the patient was sedated under local anesthesia, followed by a humpectomy and septal extension graft using harvested septal cartilage. No complications were observed during the follow-up period, and the postoperative photographs after four months showed an aesthetically sound result with no recurrence (Fig. 4).

\section{DISCUSSION}

Internal continuous osteotomy, which typically requires precision and manual dexterity, has been used conventionally for many years. This technique can produce consistent movement of the bony nasal pyramid; however, the internal continuous method is limited by complications such as bleeding, edema, and ecchymosis that are consequences of mucoperiosteal and nasal mucosal tearing $[1,3,5]$. Furthermore, if severe, an elongation of the fracture line to the base of skull can occur by unintended unfavorable fracture.

Rohrich et al. [1] concluded that the external perforating method provides excellent control and a decrease in intranasal complications such as bleeding, edema, and ecchymosis compared to the internal continuous technique; furthermore, bony stump stability is attained through the preservation of the periosteum $[1,2,10]$. In terms of disadvantages, the external method is limited by blind manipulation, the nature of its outcome is dependent upon surgical experience and intuition [11], and it can produce an external visible scar [12]. The two aforementioned techniques are blind techniques, which allow only limited direct visualization of the surgical field. Therefore, unwanted fracture or complications by hemorrhage owing to inadvertent nasal mucosal tearing can occur [1].

In light of this, we performed a subperiosteal wide dissection that was more lateral than the intended osteotomy by about 2-3 $\mathrm{mm}$ - just enough to secure direct visualization of the operative field, preserving $25 \%-30 \%$ of the nasal periosteum. With a sufficient field of vision, we therefore diagnosed the nasal deformity precisely, performed an accurate lateral osteotomy, and confirmed that an exactly complete fracture was made with the intranasal mucosa remaining uninjured, in contrast with conventional techniques. In previous studies $[1,3,13]$, the periosteum had to be preserved to prevent the growth of dead space and to maintain a more stable bony vault after surgery. However, we have tried to conduct more precise, complete intended osteotomy such that secured visualization of the surgical field was essential, so wide periosteal dissection needed to be performed. Gryskiewicz and Gryskiewicz [3] has argued that in comparing the transnasal perforating method with the internal continuous method, the perforating method was significantly more favorable, especially for surgeons with limited experience in the technique [1]. Moreover, Becker et al. [7] concluded that a large, curved, and guarded osteotome could cause considerable mucosal damage. To minimize complications and concerns due to periosteal elevation, we exploited an internal perforating method, which allowed us to make a more controlled, accurate, and complete fracture with less damage to the intranasal mucosa than with the internal continuous technique. Therefore, we carefully preserved the internal nasal mucosa and confirmed that it was intact, so that the mucosa could act as a splint for the bony tissue and compensate for bony instability due to periosteal dissection, with an internal perforating method under direct vision. In addition, the appropriate postoperative compression dressing and a Silastic angio-needle catheter for internal drainage were used to reduce dead space, which helped to prevent complications. Wide periosteal dissection offers not only better access and an enhanced visual field during the operation compared with those of conventional techniques, but it also avoids damaging the adjacent vasculature. Despite concerns over bleeding from extensive dissection, the outcomes showed a minimal incidence of ecchymosis and a limited amount of swelling; it seems that manipulation under direct vision minimizes the potential for such complications, including hemorrhage, occurring. A decrease in the risk of such complications, in turn, allowed the authors to use local anesthesia instead of general anesthesia. Furthermore, there was no need to worry about the formation of noticeable external scars. These advantages allow surgeons with little experience performing rhinoplasty to do so easily, with a reduction in operating time, allowing the patient to return to normal life more quickly.

Some argue that the wide elevation of the skin and periosteal envelope creates the potential for postoperative soft-tissue contraction, resulting in a contracted nose. However, if the fracture is confined to the lateral nasal wall, the overall nasal framework 
will be intact and can endure contracting force in the cephalic direction. Second, a thorough release of the nasal envelope allowed us to redrape the soft tissue caudally. Third, East Asians tend to undergo augmentation rhinoplasty to project the nasal profile, typically with a septal extension graft. The engagement of the skin envelope through the use of a septal extension graft helped to prevent contraction of the nose. We have not observed any cases of contracted nose formation up to the present.

Some insist that these types of procedures could have a negative impact on other accompanying procedures such as augmentation or tip plasty. However, because the authors were able to precisely determine the suitable width of midline nasal dorsum support on the basis of intact bony and cartilage nasal framework stability, along with an intact internal nasal mucosal lining, performing other procedures like augmentation with a silicone implant did not introduce problems [14]. However, further studies with larger patient groups will be needed to show that our findings on the low incidence of complications are generalizable.

In conclusion, our internal perforating method with wide periosteal dissection for lateral osteotomy surgery provides the surgeon with improved visualization of the surgical field, and we found that this method was both accurate and safe. Additionally, this method allows surgeons to carry out the preoperative plan accurately without injuring important anatomical structures, because the position of the instruments can be visualized on site. This technique also allows inexperienced surgeons to perform lateral osteotomy with ease, and thus to reduce the surgical duration; therefore, a low incidence of complications associated with hemorrhage is possible. From our experience, this novel concept will allow surgeons to perform lateral osteotomy with better outcomes and a low incidence of complications.

\section{REFERENCES}

1. Rohrich RJ, Minoli JJ, Adams WP, et al. The lateral nasal osteotomy in rhinoplasty: an anatomic endoscopic comparison of the external versus the internal approach. Plast Reconstr Surg 1997;99:1309-12.
2. Giacomarra V, Russolo M, Arnez ZM, et al. External osteotomy in rhinoplasty. Laryngoscope 2001;111:433-8.

3. Gryskiewicz JM, Gryskiewicz KM. Nasal osteotomies: a clinical comparison of the perforating methods versus the continuous technique. Plast Reconstr Surg 2004;113:1445-56.

4. Most SP, Murakami CS. A modern approach to nasal osteotomies. Facial Plast Surg Clin North Am 2005;13:85-92.

5. Yucel OT. Which type of osteotomy for edema and ecchymosis: external or internal? Ann Plast Surg 2005;55:587-90.

6. Sinha V, Gupta D, More Y, et al. External vs. internal osteotomy in rhinoplasty. Indian J Otolaryngol Head Neck Surg 2007;59:9-12.

7. Becker DG, McLaughlin RB Jr, Loevner LA, et al. The lateral osteotomy in rhinoplasty: clinical and radiographic rationale for osteotome selection. Plast Reconstr Surg 2000;105: 1806-16.

8. Cochran CS, Landecker A. Prevention and management of rhinoplasty complications. Plast Reconstr Surg 2008;122: 60e-67e.

9. Rettinger G. Risks and complications in rhinoplasty. GMS Curr Top Otorhinolaryngol Head Neck Surg 2007;6:Doc08.

10. Rohrich RJ, Janis JE, Adams WP, et al. An update on the lateral nasal osteotomy in rhinoplasty: an anatomic endoscopic comparison of the external versus the internal approach. Plast Reconstr Surg 2003;111:2461-2.

11. Rohrich RJ, Krueger JK, Adams WP Jr, et al. Achieving consistency in the lateral nasal osteotomy during rhinoplasty: an external perforated technique. Plast Reconstr Surg 2001; 108:2122-30.

12. Gryskiewicz JM. Visible scars from percutaneous osteotomies. Plast Reconstr Surg 2005;116:1771-5.

13. Ford CN, Battaglia DG, Gentry LR. Preservation of periosteal attachment in lateral osteotomy. Ann Plast Surg 1984; 13:107-11.

14. Lee YJ, Hong SH, Hong SE. Modified narrowing corrective rhinoplasty \& augmention rhinoplasty in patient with wide nasal bone. J Korean Soc Plast Reconstr Surg 2005;32:3742. 